\title{
tic\&société
}

Vol. 14, $\mathbf{N}^{\circ}$ 1-2 | 1er semestre 2020 - 2ème semestre 2020

Mutations numériques de la musique: des

contradictions à analyser

\section{Mutations numériques de la musique : des contradictions à analyser}

Introduction

Digital mutations of music: contradictions for analysis

Mutaciones digitales de la música: contradicciones que merecen ser analizadas

Christophe MAGIS et Lucien PERTICOZ

\section{(2) OpenEdition}

Journals

Édition électronique

URL : http://journals.openedition.org/ticetsociete/4658

DOI : 10.4000/ticetsociete.4658

Éditeur

Association ARTIC

Édition imprimée

Pagination : 5-11

Référence électronique

Christophe MAGIS et Lucien PERTICOZ, « Mutations numériques de la musique : des contradictions à analyser », tic\&société [En ligne], Vol. 14, № 1-2 I 1er semestre 2020 - 2ème semestre 2020, mis en ligne le 11 novembre 2020, consulté le 24 février 2021. URL : http://journals.openedition.org/ ticetsociete/4658; DOI : https://doi.org/10.4000/ticetsociete.4658 
tic\&société - 14(1-2), 2020

\title{
Introduction
}

\section{Mutations numériques de la musique : des contradictions à analyser}

\author{
Christophe MAGIS et Lucien PERTICOZ
}

Première des industries culturelles à avoir fait les frais de son manque d'adaptation au virage numérique, dès le tournant du XXIème siècle, l'industrie de la musique a, notamment au début des années 2000 , été l'objet de bon nombre d'études, dont l'essentiel a cherché à documenter la crise de la vente des supports physiques, à investir théoriquement l'essoufflement d'un modèle socioéconomique centré sur le paiement à la pièce d'un bien culturel hérité de la vente de partitions papier, voire à prophétiser l'avènement d'un nouvel essor, impulsé par les outils du web collaboratif, par le recentrage sur la gestion des droits ou par l'émergence de tel ou tel artiste à l'international. Puis ce mouvement de recherche sur le présent et le futur de l'économie de la production musicale et son articulation au numérique s'est quelque peu essoufflé, à partir du milieu des années 2010, les économistes de la culture s'intéressant davantage aux nouveaux enjeux rencontrés par d'autres filières comme le cinéma, la télévision ou le livre et laissant, pour la musique, la place à d'autres recherches, plutôt centrées sur les transformations des pratiques d'écoutes musicales.

De fait, bien au-delà de la seule dimension économique, le secteur musical a été, depuis une vingtaine d'années, le lieu d'une myriade d'expérimentations de toutes sortes autour du numérique. Qu'il s'agisse, sur le plan économique, d'une redistribution des cartes entre les divers acteurs de la filière voire l'irruption même de nouveaux acteurs, issus du web par exemple -, ou, sur le plan artistique, d'une réinvention des manières de produire la musique, de l'enregistrer, de la mixer, ou encore, sur le plan des usages, de la transformation des modalités d'accès, des modes d'écoute et même des modes d'apprentissage des instruments, c'est l'entièreté du secteur qui 
Introduction. Mutations numériques de la musique : des contradictions à analyser

s'est transformé et continue de se transformer. Cette livraison de la revue tic\&société se propose de revenir sur l'analyse de ces différentes transformations.

Bien sûr, certaines de ces évolutions ne sont ni tout à fait inédites ni tout à fait impulsées directement par le virage numérique. Métaphore localisée du fonctionnement général du capitalisme, l'industrie de la musique, connue pour sa sensibilité aux évolutions tant technologiques que sociales et culturelles ou aux transformations des usages comme des esthétiques, a une histoire ponctuée de crises régulières qui sont chaque fois l'occasion d'une réorganisation des acteurs socioéconomiques, d'une ouverture esthétique ou d'une avancée technologique à partir desquelles le secteur se réorganise afin de provoquer une nouvelle période de croissance (Bourreau et Labarthe-Piol, 2004 ; Vandiedonck, 2007). L'oubli du temps long étant souvent, dans les travaux sur la communication, et a fortiori sur les industries culturelles, l'occasion de discours tantôt alarmistes tantôt utopiques, mais dans les deux cas en général inopérants, le présent numéro entend remettre en perspective les transformations actuelles. C'est la raison pour laquelle nous avons choisi à la fois d'y intégrer des travaux issus de recherches empiriques en cours et des contributions proposant des synthèses théoriques plus générales. II sera organisé en trois temps, après une première revue de littérature qui présentera, notamment, un état des lieux ainsi que les perspectives d'une critique marxiste des mutations de l'industrie musicale - peutêtre l'un des principaux angles morts des recherches récentes sur le sujet.

Une première partie concerne l'analyse continue des évolutions économiques du secteur musical. Les deux articles qui s'y insèrent entendent investir l'actualité des stratégies de valorisation marchande de la musique et ce que celles-ci révèlent de la dimension sociopolitique des mutations en cours.

Ainsi, l'article de Guylaine Guéraud-Pinet commence par étudier l'importance que revêt, depuis la crise du tournant des années 2000, un " marché dérivé » de la musique : celui de la sonorisation télévisuelle. Une des conséquences importantes de la crise a, en effet, été de rappeler que l'industrie de la musique ne se limite pas au seul secteur de la production de phonogrammes. L'habitude prise par les commentateurs, tant journalistes qu'universitaires, d'identifier l'une à l'autre résulte 


\section{Christophe MAGIS et Lucien PERTICOZ}

d'une stratégie discursive et terminologique de l'industrie du disque bien documentée (Harker, 1997 ; Williamson et Cloonan, 2007) qui a notamment été essentielle durant la crise, permettant de donner à une baisse de la vente des supports l'illusion d'un fléau touchant le secteur musical tout entier. Avec la crise, plusieurs stratégies au sein de la filière ont concerné le recentrage sur la gestion de catalogues de droits éditoriaux et phonographiques et la diversification des activités associées de production musicale comme production intermédiaire à destination d'autres secteurs culturels. La sonorisation télévisuelle en est un cas emblématique: pratique courante depuis les débuts de la télévision, elle a peu à peu pris une importance stratégique, se voyant, depuis le début des années 2000, au cœur de stratégies croisées de diversification et de réintégration des acteurs de la télévision comme de l'industrie phonographique. Insérant son approche dans le cadre des travaux sur les mutations des industries culturelles, Guylaine Guéraud-Pinet propose de mettre au jour ces stratégies en identifiant les acteurs qui les mettent en place et en s'interrogeant sur leurs conséquences possibles quant à l'appauvrissement de la création musicale pour l'audiovisuel.

De son côté, Guillaume Heuguet étudie dans son article l'incidence des logiques de valorisation musicale d'un acteur sur la réorganisation du secteur. Initialement extérieure à l'industrie musicale, la plateforme YouTube est, en effet, devenue en une dizaine d'années la " "première destination" des auditeurs de musique en France ». Initialement positionné sur la mise à disposition d'un site permettant la publication de «blogues vidéo » par les internautes, YouTube a rapidement connu le succès en hébergeant sans rétribution des vidéos mises en ligne par des usagers contenant des œuvres musicales soumises au droit d'auteur. Son rachat par Google en 2006 a conduit à la mise en place progressive d'un modèle d'affaires intégrant la publicité et a permis la "monétisation » progressive des productions musicales publiées sur la plateforme en fonction de parts d'audience mesurées de manière nouvelle : les « vues ». C'est à l'analyse sociosémiotique précise de cette catégorie de "vue » que Guillaume Heuguet s'emploie. Articulation d'activités et acceptions multiples, faisant converger «la fantasmagorie du chiffre et du succès avec les cultures professionnelles de la télévision, du marketing et de l'informatique ", le calcul des vues, au cœur de la marchandisation de la musique sur la plateforme, 
Introduction. Mutations numériques de la musique : des contradictions à analyser

révèle une acception nouvelle de la valeur culturelle et sociale de la musique dans laquelle celle-ci est subordonnée à ses associations visuelles.

Ainsi que ces premiers textes commencent à le révéler, les transformations économiques de la filière musicale et l'émergence de nouveaux acteurs issus du web n'ont pas été sans incidence sur les pratiques professionnelles des musiciens et des créateurs de musique. Une seconde partie s'occupe alors davantage d'étudier ces incidences.

La contribution de Stéphane Costantini interroge dans ce contexte les mutations du métier de musicien et la place grandissante occupée par les compétences communicationnelles dans leurs pratiques, mais également les tactiques qu'ils mettent en oeuvre pour intégrer ces compétences sans pour autant renier leurs ambitions artistiques. Entre soumission aux injonctions à la visibilité médiatisée portées par les plateformes numériques, qui réduisent «les signes de reconnaissance artistique aux métriques et aux compteurs relationnels " - comme les « vues » analysées dans l'article précédent -, et une certaine prise de distance critique vis-à-vis de ces dispositifs, les musiciens interrogés deviennent progressivement, avec plus ou moins d'enthousiasme, des gestionnaires de leur communauté et de leur notoriété en ligne. En endossant des fonctions communicationnelles auparavant assurées par les éditeurs-producteurs, ils déchargent de fait ces derniers d'une partie de leur financement, tout en s'inscrivant pleinement dans le cadre (économique et communicationnel) délimité par les grands acteurs du numérique. Par ailleurs, la distribution inégale des compétences nécessaires pour assurer cette visibilité médiatisée participe également d'une accentuation de la concurrence et des inégalités entre les musiciens euxmêmes. Dès lors, l'auteur en conclut que c'est bien à une précarisation accrue des créateurs que ces évolutions semblent contribuer.

À la lecture de l'article de Sylvain Martet, Martin Lussier et Anouk Bélanger, ces injonctions à la visibilité médiatisée paraissent également se doubler d'une exigence d'autoformation tout au long de la carrière musicale, une exigence qui semble elle-même pleinement intériorisée par les professionnels de la musique montréalais interrogés par les auteurs. Depuis la fin des années 1970, le Québec a ainsi connu une augmentation et une 


\section{Christophe MAGIS et Lucien PERTICOZ}

diversification très nettes de l'offre institutionnelle de formations professionnalisantes visant "l'acquisition d'un ensemble d'aptitudes sociales " ou de "savoirs tacites" propres aux métiers de la musique. Toutefois, les travailleurs musicaux de leur corpus estiment que ces formations ne répondent que très partiellement aux besoins de professionnalisation et de constant renouvellement des compétences qu'engendrerait l'usage d'outils numériques de production, mais également de promotion. L'article met ainsi au jour tout un registre de discours et de pratiques qui valorisent davantage le réseautage avec les pairs et le recours à des ressources numériques en ligne. Les auteurs en concluent que la formation aux métiers de la musique est "désormais éclatée, individualisée et continue ", une tendance dont on peut se demander si elle ne contribue pas à la précarisation accrue des travailleurs musicaux dont les compétences acquises deviennent rapidement obsolètes, nécessitant dès lors un effort constant d'autoformation afin d'éviter une forme de déclassement.

Une troisième partie, enfin, s'interroge sur les usages de la musique à l'heure numérique, notamment sur les transformations des imaginaires associés aux formes d'accès à des contenus musicaux ou dérivés. Les deux articles qui la composent, chacun jalons intermédiaires de deux work in progress spécifiques, investissent ainsi la problématique générale de la "numérimorphose " des pratiques musicales (Granjon et Combès, 2007) et de ses évolutions.

L'article de Raphaël Roth et Laure-Hélène Swinnen aborde également la thématique de l'autoformation. En analysant une chaîne YouTube proposant des tutoriels gratuits pour apprendre la guitare, "MrGalagomusic », les auteurs proposent d'investir d'une manière nouvelle le problème de la gratuité des contenus à l'heure numérique et la question de son rapport à la professionnalisation de leurs créateurs. Ici, ce n'est pas directement le cas des auteurs ou des interprètes qui est analysé, mais celui des professeurs de musique. En s'appuyant sur une enquête approfondie auprès du professeur de guitare créateur de cette chaîne thématique sur YouTube, les auteurs mettent en avant les articulations complexes entre ses différentes activités de création de « tutos » gratuits, de vente de " packs premium » aux intéressés permettant d'accéder à des vidéos plus complètes, d'auteur d'une méthode de guitare 
Introduction. Mutations numériques de la musique : des contradictions à analyser

publiée aux éditions Paul Beuscher et de professeur particulier de guitare. Le rapport entre ces activités et, notamment, la difficulté de traduire le succès des " tutos " gratuits en revenus, que ce soit du côté de la monétisation des vidéos comme du côté du succès des cours à domicile, sont étudiés comme autant de " contradictions » liées aux transformations numériques des pratiques musicales.

Pour leur part, Romuald Jamet et Jonathan Roberge analysent, par une question d'apparence provocatrice (« La musique québécoise est-elle compatible avec le streaming? »), les rapports subtils qu'entretiennent les Québécois tant avec la musique francophone qu'avec l'utilisation des plateformes de streaming et la complexité de l'articulation des deux. II s'agit d'investir une situation qui semble au premier abord " paradoxale » : tandis que le streaming musical a stabilisé ses modèles socioéconomiques et participe d'un rebond de l'économie de l'industrie musicale depuis le milieu des années 2010, les artistes québécois n'en profitent guère, leur taux d'exposition et d'écoute étant faible sur les plateformes, tandis que, dans le même temps, " les Québécois affirment leur attachement à la musique et aux artistes francophones locaux ». À partir de l'analyse d'un corpus d'entretiens réalisés auprès d'auditeurs francophones au Québec, les auteurs avancent plusieurs propositions permettant d'expliquer cette apparente contradiction par une réflexion sur les usages culturels à l'ère numérique. Outre que les auditeurs interrogés sont souvent confrontés au manque d'intérêt des plateformes pour les spécificités culturelles de la musique québécoise, limitant de facto la visibilité de celle-ci (faible qualité d'indexation, manque de finesse de la recommandation...), les entretiens menés auprès d'eux révèlent également une intégration de la musique québécoise à un imaginaire d'écoute peu compatible avec les activités pendant lesquelles ils pratiquent l'écoute musicale par le streaming.

Enfin, ce numéro thématique se conclut sur un texte de Philippe Le Guern qui, au sein de la recherche francophone sur les mutations de la musique en régime numérique, figure très certainement parmi ceux qui ont apporté une des plus importantes contributions. C'est à ce titre que l'auteur se propose de brosser une synthèse personnelle de quelque vingt années de recherche sur ces questions. II y interroge le potentiel 


\section{Christophe MAGIS et Lucien PERTICOZ}

réellement « disruptif » de "l'irruption du digital dans le champ de la musique [...] par rapport à [...] [son] ère analogique ", mais également l'existence même d'un "régime prénumérique [...] auquel succéderait un régime numérique », qui aboutirait dès lors à un dépassement du fait musical même. Remontant jusqu'au début des années 1980, période de l'invention du CD et de la norme Midi, Philippe Le Guern entame un dialogue entre ses propres travaux et ceux d'autres auteurs spécialistes du sujet. II dresse ainsi un panorama des changements profonds que le passage de l'expérience musicale en régime numérique a impulsés, que ce soit sur les plans de la production, de la diffusion ou de la consommation de musique. Se mettant à bonne distance de toute posture déterministe, il élargit le spectre de sa contribution en s'interrogeant sur les implications proprement anthropologiques de cette musimorphose, une musimorphose dont le potentiel « mutagène » ne se serait, selon lui, pas encore pleinement exprimé. L'auteur conclut enfin sur une question importante qu'il laisse volontairement ouverte à la discussion : ces changements que nous observons à tous les niveaux de l'expérience musicale sont-ils finalement souhaitables ? Gageons que ce numéro de tic\&société offrira au lecteur quelques modestes éléments de réponse.

\section{Références}

Bourreau, M. et Labarthe-Piol, B. (2004). Le peer to peer et la crise de l'industrie du disque: une perspective historique. Réseaux, (125), 17-54.

Granjon, F. et Combes, C. (2007). La numérimorphose des pratiques de consommation musicale: le cas de jeunes amateurs. Réseaux, (145-146), 291-334.

Harker, D. (1997). The wonderful world of IFPI: Music industry rhetoric, the critics and the classical Marxist critique. Popular Music, 16(1), 45-79.

Vandiedonck, D. (2007). L'industrie de la musique enregistrée recomposée. Dans P. Bouquillion et Y. Combès (dir.), Les industries de la culture et de la communication en mutation (p. 91-98). Paris, France : L'Harmattan.

Williamson, J. et Cloonan, M. (2007). Rethinking the music industry. Popular Music, 26(2), 305-322. 